\title{
Pacific
}

Journal of

Mathematics

\section{ON NORMALITY OF THE CLOSURE OF A GENERIC TORUS} ORBIT IN $G / P$

\author{
ROMUALD DABROWSKI
}

Volume 172 No. 2 


\title{
ON NORMALITY OF THE CLOSURE OF A GENERIC TORUS ORBIT IN $G / P$
}

\author{
ROMUALD DABROWSKI
}

\begin{abstract}
In this paper we consider generic orbits for the action of a maximal torus $T$ in a connected semisimple algebraic group $G$ on the generalized flag variety $G / P$, where $P$ is a parabolic subgroup of $G$ containing $T$. The union of all generic $T$-orbits is an open dense (possibly proper, if $P$ is not a Borel subgroup) subset of the intersection of the big cells in $G / P$. We prove that the closure of a generic $T$-orbit in $G / P$ is a normal equivariant $T$-embedding (whose fan we explicitely describe). Moreover, the closures of any two generic $T$-orbits are isomorphic as equivariant $T$-embeddings.
\end{abstract}

\section{Introduction.}

Let $G$ be a connected semisimple algebraic group over an algebraically closed field $k$ of arbitrary characteristic. As usual, let $B^{+}$denote a fixed Borel subgroup of $G, T$ a maximal torus in $B^{+}, \Gamma(T)$ the character group of $T, B$ the opposite to $B^{+}, \Phi$ the corresponding root system in an euclidian space $(E,()),, \Phi_{+}$the set of positive roots relative to $B^{+}, \Delta$ the set of simple roots in $\Phi_{+}, s_{\alpha}$ the reflection about the linear subspace of $E$ perpendicular to root $\alpha, W$ the Weyl group of $\Phi$ generated by the reflections $s_{\alpha}, \alpha \in \Phi_{+}$ ( $W$ can also be naturally identified with $N_{G}(T) / T$ ), and $R$ the root lattice in $E$.

Let $P$ be a fixed parabolic subgroup containing $B$. Let $\Delta_{P}$ be the set of simple roots $\alpha$ such that $s_{\alpha} \in W_{P}=N_{P}(T) / T$. Then the map $P \rightarrow \Delta_{P}$ is a bijection between the set of all parabolic subgroups containing $B$ and the power set of $\Delta$ (see e.g. [B, Proposition 14.18]). We denote by $S^{P}$ the subsemigroup of the root lattice generated by all positive roots which are not sums of simple roots in $\Delta_{P}$.

We will be concerned with $T$-orbits of points in the projective variety $G / P$. Let $\lambda$ be an integral dominant weight (with respect to $\Phi_{+}$) whose stabilizer in $W$ is $W_{P}$ Then $\lambda$ extends to a character of $P$ (we will also call it $\lambda$ ), inducing a line bundle $\mathcal{L}^{\lambda}$ on $G / P$. We let $V(\lambda)$ denote the Weyl $G$-module

$$
H^{0}\left(G / P, \mathcal{L}^{\lambda}\right)=\left\{f \in k[G] \mid f(x y)=\lambda^{-1}(y) f(x) \text { for all } x \in G, y \in P\right\}
$$


of global sections of $\mathcal{L}^{\lambda}$ (see e.g. [J, Sec. 5.8, p. 84]).

Let $\Pi_{\lambda}$ denote the set of weights of $V(\lambda)$ for the action of $T$. Let $\mathcal{A}_{\lambda}$ denote the set of weights of $V(\lambda)$ listed with multiplicity. For each $\mu \in \mathcal{A}_{\lambda}$, we pick a corresponding weight vector (function) $f_{\mu}$ so that $\left\{f_{\mu} \mid \mu \in \mathcal{A}_{\lambda}\right\}$ is a basis of $V(\lambda)$. Functions $f_{\mu}, \mu \in \mathcal{A}_{\lambda}$, are called the Plücker coordinates in $G / P$. By abuse of language we use $f_{\mu}$ to denote any Plücker coordinate of a given weight $\mu$. Let $x=u . P$ be an element of $G / P$. We let $\Pi_{\lambda}(x)$ denote the set of weights $\mu \in \Pi_{\lambda}$ such that at least one of the Plücker coordinates $f_{\mu}$ does not vanish at $u$. It is easy to see that $\Pi_{\lambda}(x)$ depends on $x$ and $\lambda$ only (not on the choice of the Plücker coordinates). It turns out that $\lambda-\Pi_{\lambda} \subseteq S^{P}$. Hence by $W$-invariance of $\Pi_{\lambda}, \lambda-w \Pi_{\lambda}(x) \subseteq S^{P}$, for any $x \in G / P$ and $w \in W$. Intuitively, a torus orbit $T x \subset G / P$ can be called generic if sufficiently many Plücker coordinates of $x$ do not vanish. The following definition makes this requirement precise.

Definition 1.1. Let $x$ be an element of $G / P$. Then the torus orbit $T x \subset G / P$ is called generic if and only if $\{w \lambda \mid w \in W\} \subseteq \Pi_{\lambda}(x)$, and for each $w \in W$, the semigroup generated by $\lambda-w \Pi_{\lambda}(x)$ is $S^{P}$ (that is, the maximal semigroup that $\lambda-w \Pi_{\lambda}(x)$ can generate).

We will show that this definition does not depend on the choice of $\lambda$. It turns out that $\Pi_{\lambda}(x)=\Pi_{\lambda}$ implies $T x$ is generic. Therefore generic orbits exist since there are points in $G / P$ at which all Plücker coordinates do not vanish. We will also prove that in the case of $G / B, T x$ is generic if and only if $x$ belongs to $\bigcap_{w \in W / W_{P}} w B^{+} . P$.

The aim of this note is to prove that the closure of a generic $T$-orbit in $G / P$ is a normal equivariant $T$-embedding. We can then use the general theory of equivariant torus embeddings (see e.g. [K, Oda1]) to show that the closures of any two generic orbits are isomorphic (as equivariant $T$-embeddings). We prove this by identifying the fan describing the isomorphism class of these $T$-embeddings.

Remark. We point out that if $P \neq B$, the definition of generic $T$-orbit given here differs from the one used in [F-H, Remark 1, p. 257]. There, an orbit $T x$ is called "generic" if and only if $x$ belongs to the non-degenerate stratum $Z=\bigcap_{w \in W / W_{P}} w B^{+} . P$ in the stratification of $G / P$ introduced in [G-S] (note that in [F-H] $B$ is the "positive" Borel subgroup, while here $B$ denotes the "negative" Borel subgroup). It is easy to see that the set of all $x \in G / P$ with $T x$ generic in the sense of Definition 1.1 is an open subset of $Z$. It is proved in [G-S, Section 5.1, Proposition 1] that if $k$ is the field of complex numbers then the image under the moment map of the closure of each torus orbit contained in $Z$ is the convex hull of $\{w \lambda \mid w \in W\}$. In [F-H] the general theory of torus embeddings is used to study the closure of $T x$ in 
$G / P$ for $x \in Z$. It appears however that normality of these varieties, required in the theory, has not been proved (as pointed out in [Oda2, Section 2.6]). Also, contrary to what is claimed in $[\mathbf{F}-\mathbf{H}]$, two $T$-orbits in $Z$ may have nonisomorphic closures in $G / P$ (see the example below).

Example. Let $\mathbf{C}$ denote the field of complex numbers. Let $q$ be a nondegenerate quadratic form on $V=\mathbf{C}^{5}$, and let $G=S O(q)$ be the subgroup of determinant one linear transformations of $V$, preserving $q$. Then $G$ is a connected, semisimple, rank 2 algebraic group over $\mathbf{C}$, and $V$ is an irreducible representation of $G$. Let $L$ be a fixed isotropic line for $q$ (that is $q(v)=0$ for all $v \in L$ ), and let $P \subset G$ be the stabilizer of $L$. Then $P$ is a parabolic subgroup of $G$, and $G / P$ is naturally isomorphic to the smooth quadric hypersurface $Q$ in the complex projective space $\operatorname{Proj}(V)$ given by the homogeneous equation $q(x)=0$. For brevity, we will equate $G / P$ with $Q$. Let $\left\{e_{1}, e_{2}, e_{3}, e_{4}, e_{4}\right\}$ be the standard basis of $V$ and let $q(x)=x_{1} x_{3}+x_{2} x_{4}-2 x_{5}^{2}$, where $\left[x_{1}, x_{2}, x_{3}, x_{4}, x_{5}\right]$ are the coordinates of $x \in V$ relative to the standard basis. We let $L=\mathbf{C} e_{1}$. Then the maximal torus contained in $P$ is $T=\left\{\operatorname{diag}\left(t_{1}, t_{2}, 1 / t_{1}, 1 / t_{2}, 1\right) \mid t_{\imath} \in \mathbf{C} \backslash\{0\}, i=1,2\right\}$. Here, the Plücker coordinates in $Q=G / P$ are just the standard homogeneous coordinates in $\operatorname{Proj}(V)$. Clearly, $L_{1}=\mathbf{C}[1,1,-1,1,0]$ and $L_{2}=\mathbf{C}[1,1,1,1,1]$ are $q$ isotropic. Also, the $T$-orbits of $L_{1}$ and $L_{2}$ are "generic" in the sense of $[\mathbf{F}-\mathbf{H}]$, but only $T L_{2}$ is generic in the sense of the Definition 1.1. Also, $\Pi\left(L_{1}\right) \neq \Pi\left(L_{2}\right)=\Pi$, where $\Pi$ denotes the set of weights of $V$. This directly contradicts Lemma 13 in $[\mathbf{F}-\mathbf{H}]$. Let $X_{i}=\overline{T L_{i}}, \quad i=1,2$, where the closure is taken in $Q$ (or in $\operatorname{Proj}(V)$, since $Q$ is closed in $\operatorname{Proj}(V))$. It is easy to see that $X_{1}$ is isomorphic to $\mathbf{C} P^{1} \times \mathbf{C} P^{1}$. On the other hand $X_{2}$ is the singular closed subvariety of $\operatorname{Proj}(V)$ given by homogeneous equations $x_{1} x_{3}=x_{5}^{2}$, $x_{2} x_{4}=x_{5}^{2}$ (the singular points of $X_{2}$ are $[1: 1: 0: 0: 0],[1: 0: 0: 1: 0]$, $[0: 1: 1: 0: 0]$, and $[0: 0: 1: 1: 0])$. Therefore, the example shows that two $T$-orbits "generic" in the sense of $[\mathbf{F}-\mathbf{H}]$ may not have isomorphic closures in $G / P$.

\section{Weights of Weyl $G$-modules.}

We will need the following notation. For any additive set $A$ of real numbers and any subset $Y$ of $E$, let $A Y$ denote the set of all linear combinations of elements in $Y$ with coefficients in $A$. By definition, a semigroup $S$ contained in a lattice $L$ in $E$ is saturated in $L$ if and only if

$$
L \cap \mathrm{Q}_{+} S=S
$$

(see [K, Chapter 1, Section 1]). Equivalently, $S$ is saturated in $L$ if and only if for any positive integer $m, m \mu \in S$ and $\mu \in L$ imply $\mu \in S$. 
Proposition 2.1. $S^{P}$ is saturated in $R$.

Proof. Let $\Phi_{+}^{P}$ denote the set of positive roots which are not linear combinations of roots in $\Delta_{P}$. Then $S^{P}=\mathrm{Z}_{+} \Phi_{+}^{P}$. Suppose that $S^{P}$ is not saturated in $R$. Let $\mu \in R$ be an element of minimal height among the elements of $Q_{+} \Phi_{+}^{P}$ which are not elements of $S^{P}$. Then $\mu=\mu_{1}+\mu_{2}$, with

$$
\mu_{1}=\sum_{\beta \in M} m_{\beta} \beta
$$

where $M \subseteq \Phi_{+}^{P}, m_{\beta}$ are positive integers, and

$$
\mu_{2}=\sum_{\alpha \in N} n_{\alpha} \alpha
$$

where $N \subseteq \Delta_{P}$, and $n_{\alpha}$ are positive integers. From the above decompositions of $\mu$ we choose one with $\mu_{2}$ of minimal height. Since the sum of any two roots with negative scalar product is again a root, minimality of $\mu_{2}$ implies that

$$
(\alpha, \beta) \geq 0,
$$

for all $\alpha \in N, \beta \in M$. Take any simple root $\alpha$ in $N$, such that $\left(\mu_{2}, \alpha\right)>0$. Consider $\nu=s_{\alpha}(\mu) \in R$. Since elements of $\Phi_{+}^{P}$ are permuted by $s_{\alpha}, \nu$ belongs to $\mathrm{Q}_{+} \Phi_{+}^{P}$ but not to $S^{P}$. This is a contradiction, since $\operatorname{ht}(\nu)<\operatorname{ht}(\mu)$ and $\mu$ was assumed to be of minimal height among the root lattice elements in $\mathrm{Q}_{+} \Phi_{+}^{P}$, not in $S^{P}$.

Let $V(\lambda), \lambda, \Pi_{\lambda}$ be as in the introduction. The following proposition lists some basic properties of $\Pi_{\lambda}$.

\section{Proposition 2.2.}

(i) $\lambda-\Pi_{\lambda}$ coincides with the set of root lattice points in the convex hull of $\{\lambda-w \lambda \mid w \in W\}$.

(ii) $S^{P}$ is generated by $\lambda-\Pi_{\lambda}$. If $P=B$ and $\lambda$ is the sum of fundamental weights then $S^{P}$ is generated by $\{\lambda-w \lambda, w \in W\}$.

Remark. Part (i) is well known, but were not able to locate an appropriate reference.

Proof. We first observe that the weights of the Weyl module $V(\lambda)$ ( $\lambda$ integral dominant) are independent of the characteristic of $k$. This follows from thefact that character formulas for Weyl modules are the same in each characteristic. Therefore we can assume, that $\operatorname{char}(k)=0$.

Part (i). Let $C$ denote the convex hull of $\{w \lambda \mid w \in W\}$ and we let $\Pi=$ $(\lambda+R) \cap C$. We have to prove that $\Pi=\Pi_{\lambda}$. It is a known fact that $\Pi_{\lambda} \subset \lambda+R$. 
Therefore it is enough to show that $\Pi_{\lambda}$ is contained in $C$. Suppose that this is not the case, and let $\mu$ be a weight in $\Pi_{\lambda}$, not in $C$. Assume also, that $\mu$ is a maximal such weight in the usual order in $E$ relative to $\Phi_{+}$. Since both $\Pi_{\lambda}$ and $C$ are $W$-invariant, we must have $s_{\alpha}(\mu) \leq \mu$ for all positive roots $\alpha$. Hence $\mu$ is dominant. Since $\mu$ is not the highest weight $\lambda$, there must be a positive root $\alpha$ and a positive integer $m$ such that $\mu_{1}=\mu+m \alpha \in \Pi_{\lambda}$. Then by maximality of $\mu, \mu_{1}$ (hence also $s_{\alpha}\left(\mu_{1}\right)$ ) is in $C$. A straightforward computation shows that $\mu$ belongs to the line segment connecting $\mu_{1}$ and $s_{\alpha}\left(\mu_{1}\right)$. This is a contradiction, since we have assumed that $\mu$ is not in $C$.

We are left with showing that $\Pi$ is contained in $\Pi_{\lambda}$. An easy argument by induction on the length function in $W$, shows that for any $w \in W, \lambda-w \lambda$ is a sum of roots in $\Phi_{+}^{P}$. Therefore $\Pi$ is contained in $\lambda-\mathbf{Z}_{+} \Phi_{+}$. It is proved in $\left[\mathbf{H}\right.$, Proposition, p. 114] that the elements of $\Pi_{\lambda}$ are exactly the weights whose $W$-orbit is contained in $\lambda-\mathbf{Z}_{+} \Phi_{+}$. Hence $\Pi \subseteq \Pi_{\lambda}$, as required.

Part (ii) We have observed in the proof of Part (i) that $\lambda-C$ is contained in convex cone spanned by $\Phi_{+}^{P}$. Therefore

$$
\lambda-\Pi_{\lambda}=R \cap(\lambda-C) \subseteq S^{P}
$$

since $S^{P}$ is saturated in $R$. The opposite inclusion holds since $\Phi_{+}^{P} \subseteq \lambda-\Pi_{\lambda}$. This follows from the fact that weigths of irreducible $G$-representations (in characteristic 0 ) satisfy the following property: for any positive root $\alpha$, and a positive integer $n$, if $\mu$ and $\mu-n \alpha$ are weights of the representation, so are $\mu-q \alpha$ for any $q, 0 \leq q \leq n$ (see e.g. [H, Sec. 21.3, Prop.]). One applies this property to $\lambda$ and $s_{\alpha}(\lambda)$, where $\alpha \in \Phi_{+}^{P}$.

The second claim of Part(ii) follows since $\Delta \subseteq\{\lambda-w \lambda \mid w \in W\}$ if $\lambda$ is the sum of fundamental weights.

\section{Generic orbits of $T$ in $G / P$.}

Let $x \in G / P$ and let $X$ denote the the closure of $T x$ in $G / P$. For any $w \in W$, let

$$
Y_{w}=\left\{y \cdot P \mid f_{w \lambda}(y) \neq 0\right\}=\left\{y \cdot P \mid w \lambda \in \Pi_{\lambda}(y \cdot P)\right\}
$$

and

$$
X_{w}=Y_{w} \cap X .
$$

It is well known that each $Y_{w}$ is an affine space which is open in $G / P$ and whose coordinate ring is generated by functions $f_{\mu} / f_{w \lambda}, \mu \in \mathcal{A}_{\lambda}$. Moreover, the union of $Y_{w}, w \in W$ is $G / P$. Let $T_{x}=\{t \in T \mid t x=x\}$ and $T^{x}=T / T_{x}$. We have the following proposition 
Proposition 3.1. Let $x \in G / P$.

(i) Tx is open in $X$ and it is isomorphic to $T^{x}$. Therefore, $X$ is an equivariant $T^{x}$-embedding in the sense of $[\mathbf{K}]$.

(ii) $\left\{X_{w} \mid w \in W, w \lambda \in \Pi_{\lambda}(x)\right\}$ is a covering of $X$ by $T$-invariant open affine subsets of $X$. The coordinate ring of $X_{w}, w \lambda \in \Pi_{\lambda}(x)$, is the subalgebra of $k\left[T^{x}\right]=k\left[\Gamma\left(T^{x}\right)\right]$ generated by $\Pi_{\lambda}(x)-w \lambda$.

(iii) Let $w \in W$ be such that $w \lambda \in \Pi(x)$. Then

$$
T_{x}=\{t \in T \mid \mu(t)=1 \text { for all } \mu \in w \lambda-\Pi(x)\} \text { 。 }
$$

Proof. The fist part of (i) follows from the fact the map $t \rightarrow t x$ is a separable morphism from $T$ onto an open subvariety $T x$ of $X$ whose fibers are the cosets of $T_{x}$ in $T$ (the morphism is separable since it is the composition of the inclusion of $T$ in $G$ with the quotient map from $G$ to $G / P$ ).

Part (ii) follows, since for each $w \in W$ such that $w \lambda \in \Pi_{\lambda}(x), X_{w}$ can be viewed as a closed $T$-invariant subvariety of the affine space $Y_{w}$. Hence the coordinate ring of $X_{w}$ is generated by the restrictions to $X_{w}$ of functions $f_{\mu} / f_{w \lambda}, \mu \in \mathcal{A}_{\lambda}$.

Part(iii). Suppose that $w \in W$ satisfies $w \lambda \in \Pi(x)$. Then $x \in X_{w}$. Clearly, $t \in T_{x}$ if and only if $t$ fixes all elements of $X_{w}$ (or equivalently, $t$ fixes all regular functions on $X_{w}$ ). Therefore the desired formula for $T_{x}$ follows from the description of the coordinate ring of $X_{w}$ given in (ii).

Before we state a corollary of Proposition 3.1, we need to introduce the following notation. Let $R^{P}$ denote the subgroup of the root lattice generated by $S^{P}$. One can show that $R^{P}=R$ if $\Phi$ is an irreducible system. If $\Phi$ a union of irreducible root systems $\Phi_{j}, j \in J$, then $R^{P}$ is the root lattice of the root system

$$
\cup\left\{\Phi_{j} \mid \Phi_{j} \cap S^{P} \neq \emptyset\right\}
$$

Let

$$
T_{P}=\bigcap_{\nu \in R^{P}} \operatorname{ker}(\nu)
$$

Note that if $R^{P}=R$, then $T_{P}$ is coincides with the center of $G$.

Corollary. (Suggested by the referee.)

(i) The stabilizer of each generic torus orbit is $T_{P}$. Moreover, $T_{P}$ it is the smallest subgroup of $T$ among the $T$-stabilizers of elements of $G / P$.

(ii) (Partial converse of (i)). If $x \in G / P$ is such that $T x$ is contained in the nondegenerate stratum $Z, \overline{T x}$ is normal and $T_{x}=T_{P}$, then $T x$ is generic. 
Proof. Part (i) follows from Proposition 3.1 (iii). Suppose that $T x$ satisfies the assumptions of (ii). Let $S^{x}$ denote the semigroup generated by $\lambda-\Pi_{\lambda}(x)$. We have to show that $S^{x}=S^{P}$. Since $T_{x}=T_{P}$, one has

$$
\bigcap_{\nu \in R^{P}} \operatorname{ker}(\nu)=\bigcap_{S^{x}} \operatorname{ker}(\nu)
$$

by Proposition 3.1 (iii). Therefore $R^{P}$ is generated by $S^{x}$ as a subgroup of $\Gamma(T)$. Assumed normality of $\overline{T x}$ implies that $S^{x}$ is saturated in $R^{P}$. On the other hand $\{\lambda-w \lambda \mid w \in W\} \subset S^{x}$ since $T x$ is assumed to be generic. Hence $S^{x}=S^{P}$ since both semigroups are saturated in $R^{P}$ and $\mathrm{Q}_{+} S^{x}=\mathrm{Q}_{+} S^{P}$ by Proposition 2.2.

From now on we assume for simplicity that $R^{P}=R$ (equivalently, $S^{P}$ contains at least one root from each irreducible component of $\Phi$ ). Let $W^{P} \subseteq$ $W$ be a fixed set of representatives of $W / W_{P}$. Let $D$ denote the fundamental chamber $\{\nu \in E \mid(\mu, \alpha) \geq 0$ for all $\alpha \in \Delta\}$. We are now ready to state the main result of this paper.

Theorem 3.2. Let $x \in G / P$ be such that $T x \subset G / P$ is generic. Let $X=\overline{T x}$. Then:

(i) $X$ is a normal variety (hence by $[\mathbf{K}$, Theorem 14, page 52], also CohenMacaulay with rational singularities).

(ii) The fan corresponding to $X$ consists of the cones

$$
C_{w}=-w \bigcup_{z \in W_{P}} z D, \quad w \in W^{P}
$$

together with their faces. In particular, the closures of any two generic orbits in $G / P$ are isomorphic as $T$-equivariant embeddings.

Proof. Part (i). By [K, Theorem 6, p. 24] a general equivariant $T$-embedding is a normal variety if and only if it admits a covering by open affine $T$-stable subvarieties whose coordinate rings are generated by semigroups saturated in $\Gamma(T)$. Hence Part(ii) follows from Propositions 3.1 and 2.1.

Part(ii) follows, since the dual cone of $S^{P}$ is $\bigcup_{z \in W_{P}} z D$, and by Proposition 3.1(ii) the coordinate ring of $X_{w}, w \in W$, is $k\left[-w S^{P}\right]$.

The following theorem shows that Definition 1.1 of a generic torus orbit does not depend on the choice of the Weyl module $V(\lambda)$.

Theorem 3.3. Let $x \in G / P$. The following statements are equivalent.

(i) There exist an integral dominant weight $\lambda$ whose stabilizer in $W$ is $W_{P}$, such that for any $w \in W$, the semigroup generated by $\lambda-w \Pi_{\lambda}(x)$ is $S^{P}$. 
(ii) For each integral dominant weight $\lambda$ whose stabilizer in $W$ is $W_{P}$, and each $w \in W$, the semigroup generated by $\lambda-w \Pi_{\lambda}(x)$ is $S^{P}$.

(iii) There exists an integral dominant weight $\lambda$ whose stabilizer in $W$ is $W_{P}$, such that $\Pi_{\lambda}(x)=\Pi_{\lambda}$.

Proof. Clearly, (ii) implies (i). Also, by Proposition 2.2, (iii) implies (i). We have to prove that if (i) holds, so do (ii) and (iii). Let $X=\overline{T x}$ and let $X_{w}, w \in W$ be as in Theorem 3.1. Since the coordinate ring of $X_{w}$ does not depend on the choice of a Plücker embedding, Theorem 3.1(ii) implies that (ii) follows from (i).

It remains to prove that (i) implies (iii). Let $x \in G / P$ and let $\lambda$ be as in (i). For any integral dominant weight $\mu$ whose stabilizer in $W$ is $W_{P}$, let $\mathcal{L}^{\mu}$ denote the corresponding line bundle on $G / P$. Let $\mathcal{L}_{X}^{\mu}$ denote the pullback of $\mathcal{L}^{\mu}$ to $X=\overline{T x}$. Since $X$ contains an open, dense $T$-orbit, every weight of $H^{0}\left(X, \mathcal{L}_{X}^{\mu}\right)$ under the natural $T$-action has multiplicity one. Therefore the dimension of the image of the restriction map

$$
H^{0}\left(G / P, \mathcal{L}^{\mu}\right) \rightarrow H^{0}\left(X, \mathcal{L}_{X}^{\mu}\right)
$$

is $\sharp\left(\Pi_{\mu}(x)\right)$. We observe that line bundle $\mathcal{L}_{X}^{\mu}$ is ample. This is because the piecewise linear function on $E$ corresponding to $\mathcal{L}_{X}^{\mu}$ (see [F-H, Theorem 2]) is strictly upper convex. Then the description of the fan of $X$ given in Theorem 3.2(iii), [Oda1, Theorem 2.13 and Corollary 2.9], and Proposition 2.2 (i) imply that

$$
\operatorname{dim} H^{0}\left(X, \mathcal{L}_{X}^{\mu}\right)=\sharp\left(\Pi_{\mu}\right) .
$$

Since $\mathcal{L}^{\lambda}$ is ample there exists a positive integer $q$ such that the restriction map

$$
H^{0}\left(G / P, \mathcal{L}^{q \lambda}\right) \rightarrow H^{0}\left(X, \mathcal{L}_{X}^{q \lambda}\right)
$$

is surjective. Hence $\Pi_{q \lambda}(x)=\Pi_{q \lambda}$ as required.

It is easy to see that Theorem 3.3 and Proposition 2.2 imply:

Corollary. Let $x \in G / B$. Then $T x$ is generic if and only if $x \in \bigcap_{w \in W} w B^{+} . B$ (i.e. it is "generic" in the sense of $[\mathbf{F}-\mathbf{H}])$. Moreover, if $x T$ is generic then $X=\overline{T x}$ is smooth.

Remark. Smoothness of the closure of a generic torus orbit in $G / B$ is well known (we do not know however, to whom this fact should be attributed).

\section{Final remarks and questions.}

1. All results about closures of $T$-orbit in $G / P$ stated in $[\mathbf{F}-\mathbf{H}]$ hold for generic orbits (in the sense of Definition 1.1) in any characteristic. This is 
because the arguments used in $[\mathbf{F}-\mathbf{H}]$ are valid for normal equivariant $T$ embeddings, and we have shown that the closure of a generic orbit is such an embedding. We do not know however, if the results remain valid for all $T$-orbits in the nondegenerate stratum if $P \neq B$.

2. Let $X$ denote the closure of a $T$-orbit of an element $x \in G / P$. It is not difficult to prove that if $\lambda$ is an integral dominant weight whose stabilizer in $W$ is $W_{P}$, then the line bundle $\mathcal{L}_{X}^{\lambda}$ is in fact very ample (one can use the criterion for very ampleness given in [F, Lemma, p. 69] or [Oda1, Corollary 2.9]). Then it follows from [F, Exercise, p. 72] that the corresponding embedding of $X$ in $\operatorname{Proj}\left(H^{0}\left(X, \mathcal{L}_{X}^{\lambda}\right)\right)$ is projectively normal and Cohen-Macaulay (that is, the homogeneous coordinate ring of $X$ in $\operatorname{Proj}\left(H^{0}\left(X, \mathcal{L}_{X}^{\lambda}\right)\right)$ is normal and Cohen-Maculay). Therefore, the embedding $X \subset \operatorname{Proj}\left(H^{0}\left(G, \mathcal{L}^{\mu}\right)\right)$ is also projectively normal and Cohen-Macaulay, if the restriction map from $H^{0}\left(G / P, \mathcal{L}^{\lambda}\right)$ to $H^{0}\left(X, \mathcal{L}_{X}^{\lambda}\right)$ is surjective (equivalently $\left.\Pi_{\lambda}(x)=\Pi_{\lambda}\right)$. We do not know if this is so, if $T x$ is generic and $\Pi_{\lambda}(x) \neq \Pi_{\lambda}$.

3. Since the closure of any $T$-orbit in an equivariant normal $T$-embedding is normal (see [K, Proposition 2, p. 17]), $X$ is normal if it is contained in the closure of a generic $T$-orbit. In this situation, the fan corresponding to $X$ can be described explicitly in terms of the fan defined in Theorem 3.2 (iii) (see e.g. [Oda2, Section 1.1]). Since there could be non-generic orbits of maximal dimension (see the example in the introduction) not every $T$-orbit is contained in the closure of a generic one. The structure of the orbit is not clear. Does it have to be normal? If yes, what is its fan? Suppose that the closures of all $T$-orbits in $G / P$ are indeed normal. Then the Example and the Corollary of Proposition 3.1, suggest the conjecture that the isomorphism type of $\overline{T x}$ (as a torus equivariant embedding) is determined by two pieces of data: the stabilizer of $x$ in $T$ and the set $\left\{w \in W / W_{P} \mid x \in B^{+} w . P\right\}$.

\section{References}

[B] N. Bourbaki, Groupes et algèbres de Lie, Ch. 4-6, Hermann, Paris, 1968.

[F] W. Fulton, Introduction to Toric Varieties, Princeton University Press, Princeton, New Jersey, 1993.

[F-H] H. Flaschka and L. Haine, Torus orbits in G/P, Pacific. J. Math., 149 (1991), 251-292.

[G-S] I.M. Gelfand V.V. Serganova, Combinatorial geometries and torus strata on homogeneous compact manifolds, Russian Math. Surveys, 42 (1987), 133-168.

[H] J.H. Humphreys, Introduction to Lie Algebras and Representation Theory SpringerVerlag, New York, 1972.

[J] J.C. Jantzen, Representations of Algebraic Groups, Academic Press, Boston, 1987.

[K] G. Kempf, F. Knudsen, D. Mumford and B. Saint-Donat, Toroidal Embeddings I, Lecture Notes in Math., 339 Springer-Verlag, Berlin-Heidelberg-New York, 1973. 
[Oda1] T. Oda, Convex bodies and algebraic geometry - an introduction to the theory of toric varieties, Springer-Verlag, Berlin-Heidelberg-New York, 1988.

[Oda2] _ Geometry of toric varieties, Proc. of the Hyderabad conference on algebraic groups, Manoj Prakashan, Madras -India, 1991.

Received September 25, 1993 and revised February 16, 1994.

Columbia University

NEW YORK, NY 10027

E-mail address: rdab@math.columbia.edu 
Rosa M. Miró-Roig, Singular moduli spaces of stable vector bundles on $\mathbf{P}^{3}$

Hitoshi Moriyoshi and Toshikazu Natsume, The Godbillon-Vey Cyclic Cocycle and Longitudinal Dirac Operators

J.C. Naranjo, The positive dimensional fibres of the Prym map

Artur Nicolau and Arne Stray, Nevanlinna's coefficients and Douglas al-

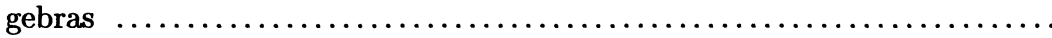

K.K. Park, Entropy of a skew product with a $Z^{2}$-action $\ldots \ldots \ldots \ldots \ldots .227$

María Cristina Pereyra, Sobolev spaces on Lipschitz curves ......... 553

T. Sano, Commuting co-commuting squares and finite dimensional Kac algebras

H.B. Thompson, Second order ordinary differential equations with fully nonlinear two point boundary conditions

H.B. Thompson, Second order ordinary differential equations with fully nonlinear two point boundary conditions II

F. $\mathrm{Xu}$, The flat part of non-flat orbifolds

Hidenobu Yoshida, A type of uniqueness for the Dirichlet problem on a half-space with continuous data 


\section{PACIFIC JOURNAL OF MATHEMATICS}

Volume $172 \quad$ No. $2 \quad$ February 1996

On the failure cycles for the quadratic normality of a projective variety

307

EdoARdo BaLlico

On the minimal free resolution of general embeddings of curves

315

EdOARDO BALlico

On normality of the closure of a generic torus orbit in $G / P$

321

ROMUALD DABROWSKI

Paragroupe d'Adrian Ocneanu et algèbre de Kac

331

MARIE-Claude DAVID

Irreducibility and dimension theorems for families of height 3 Gorenstein algebras

SuSAN J. DiESEL

On the cohomology of the Lie algebra $L_{2}$

ALICE FIALOWSKI

Generic differentiability of convex functions on the dual of a Banach space

John R. Giles, P. S. Kenderov, WarRen Brian Moors and S. D.

SCIFFER

Moon hypersurfaces and some related existence results of capillary hypersurfaces

without gravity and of rotational symmetry

FEI-TSEN LIANG

Stable relations. II. Corona semiprojectivity and dimension-drop $C^{*}$-algebras

TERRY ATHERTON LORING

Singular moduli spaces of stable vector bundles on $\mathbf{P}^{3}$

Rosa M. MIRó-ROIG

The Godbillon-Vey cyclic cocycle and longitudinal Dirac operators

Hitoshi MoRIYOSHI and TOSHIKAZU NATSUME

Nevanlinna's coefficients and Douglas algebras

ARTUR NiCOLAU and ARNE STRAY

Sobolev spaces on Lipschitz curves

MARÍA CRISTINA PEREYRA

A type of uniqueness for the Dirichlet problem on a half-space with continuous data 